\title{
Noise characteristic analysis and sound sources identification for rod-airfoil interaction using different subgrid-scale models
}

\author{
Chenghao Yang ${ }^{1}$, Heying Feng ${ }^{1 *}$, and Yehui Peng ${ }^{2}$ \\ ${ }^{1}$ Hunan Provincial Key Laboratory of Health Maintenance for Mechanical Equipment, Hunan University of Science and Technology, \\ Xiang tan, Hunan, 411201, China \\ ${ }^{2}$ School of Mathematics and Computational Science, Hunan University of Science and Technology, Xiang tan, Hunan, 411201, China
}

\begin{abstract}
Four subgrid-scale models based on large eddy simulation (LES), such as Smagorinsky-Lilly (SL), dynamic Smagorinsky-Lilly (DSL), wall-adapting local eddy-viscosity (WALE), and dynamic kinetic-energy transport (KET) were used and couple Ffowcs Williams-Hawkings equation to accurately analyze and identify the characteristics and position of the sound sources of rod-airfoil interaction. The results of four models were compared with experimental data. It was found that the DSL model was the optimal subgrid-scale model for the study of the interaction noise considering the calculation accuracy. Therefore, the DSL model was selected for analyzing and identifying the characteristics and location of the interaction noise source. During the calculation, solid and permeable data surfaces were used for acoustic integral surfaces. The results show that the impact of the quadrupole source is negligible at a low Mach number, and the dipole noise coming from the pressure fluctuations is dominant. Meanwhile, the dipole noise from the airfoil is louder than that from the rod; the leading edge of about $30 \%$ chord length of airfoil the is the main sound source of interference effect. Above results can provide guidance for research of blade-vortex interaction noise.
\end{abstract}

\section{Introduction}

The noise generated by the mutual interference effect between upstream wakes and downstream blades, such as aircraft engine rotor/stator interaction noise, ventilator, helicopter blade-vortex interaction noise, etc., is a common phenomenon in the industrial field [1]. Owing to its simple structure, the rod-airfoil model is considered a simplified interaction model for investigating rotor/stator interaction noise [2]. Currently, there are several widely used subgrid-scale models, such as Smagorinsky-Lilly (SL), dynamic Smagorinsky-Lilly (DSL), wall-adapting local eddy-viscosity (WALE), and dynamic kinetic-energy transport (KET). Different subgrid-scale models will result in different flow details, and the accuracy of acoustic far-field depend on the flow field. Although many scholars studied the influence of different subgrid-scale models on flow field details [3-6], there are few comparative studies on different subgridscale models of the accuracy for interaction noise. Therefore, it is necessary to select the most suitable subgrid-scale model in the follow-up study on noise characteristics and sound identification. Regarding the influence of quadrupole noise under low Mach, the number conclusions obtained in different literatures are not consistent $[6,7]$.

Based on the above analysis, we compare and analyze the results of the flow field, noise spectrum and corresponding experimental data, and then select a more accurate subgrid-scale model. Furthermore, we identify sound sources, analyze the influence of the quadrupole source at low Mach number, and obtain more accurate values and positions of noise sources.

\section{Methodology}

In this study, LES method coupling Ffowcs WilliamsHawkings (FW-H) equation is used to study the rodairfoil interaction noise. The LES method separates large-scale from small-scale vortexes in the flow field by the filtering function and directly calculates the largescale vortexes using the $\mathrm{N}-\mathrm{S}$ equation. The influence of small-scale vortexes on large-scale vortexes is simulated by the turbulence model, referred to as the subgrid-scale model. In this study four subgrid-scale models: SL model, DSL model, WALE model, and KET model are used. The numerical simulations results were verified by comparing them with the experimental data by Jocob et al. [8]. The experiment setup of rod-airfoil interaction wind tunnel is shown in Figure 1.

Figure 2 shows the computational domain. The airfoil, chord length of the airfoil, and rod diameter are NACA0012, $c=100 \mathrm{~mm}$, and $d=10 \mathrm{~mm}$, respectively. The $k-\omega$ SST turbulence model was used for a steady solution, and then the results were used as the initial solution for LES. The time step size was set to $2 \times 10^{-6} \mathrm{~s}$. The boundary conditions and parameters are based on

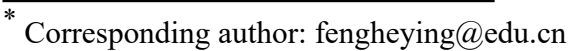


experimental conditions. The velocity of inlet is set to 72 $\mathrm{m} / \mathrm{s}$, and its turbulence is set to $0.78 \%$. The outlet is set to the pressure outlet boundary, whereas the static pressure is $98.9 \mathrm{KPa}$. The spanwise direction of the computational domain is set to a translational periodic boundary condition.

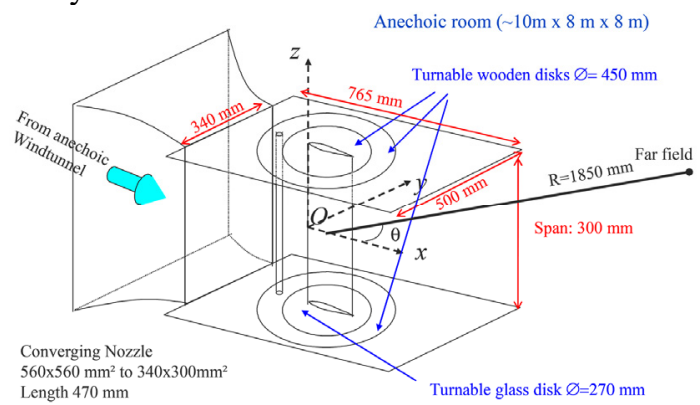

Fig. 1. The experimental setup of rod-airfoil.

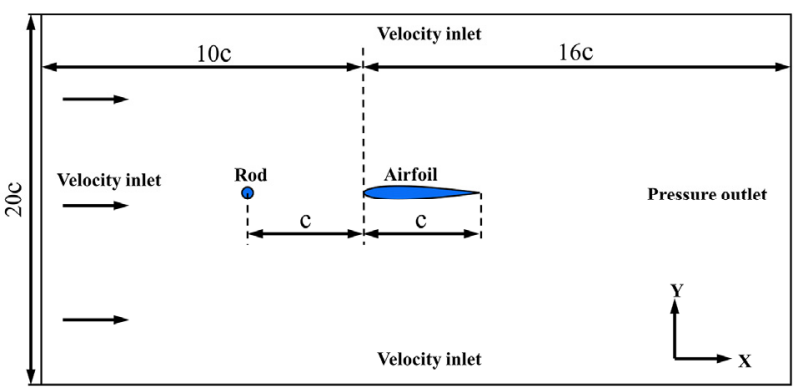

Fig. 2. Computational domain.

\section{Results and discussion}

The time histories of lift and drag coefficients for rod and airfoil by different subgrid-scale models are plotted in Figure 3. Figures 3 (a) - (d) present results of SL, DSL, WALE, and KET models, respectively. It can be observed that the rod drag coefficient in the DSL, WALE, and KET models fluctuate around 1, whereas the rod drag coefficient of the SL model is over predicted by about $30 \%$ during the time step of $10,000-20,000$. Concurrently, the rod lift coefficient with this model shows a large fluctuation in the middle time period, and the fluctuation in the beginning and end stages is small. Therefore, the periodic fluctuation has poor symmetry in the whole stage. And the rod lift coefficient by the WALE model is relatively small throughout the whole stage because the model coefficient is fixed, resulting in a large dissipation of the model. The results of lift and drag coefficient of the SL and WALE models reflected on the spectrum, causing vortex shedding frequency error. Generally, the results of the DSL model are the most ideal, with the lift/drag coefficient showing good symmetry for the entire time domain.

Next, the error value between the vortex shedding frequency obtained by fast Fourier transform and the experimental value will be compared in Table 1. The Strouhal number of vortex shedding is 0.192 in experimental result. The result of DSL model is in good agreement with the actual value, with minimum errors of $0.99 \%$. The vortex shedding frequency of WALE model is overpredicted by approximately $8.39 \%$. This may be because WALE is an algebraic model, and its model constants are unchanged; thus, it cannot perform secondary filtering like the DSL subgrid-scale model.

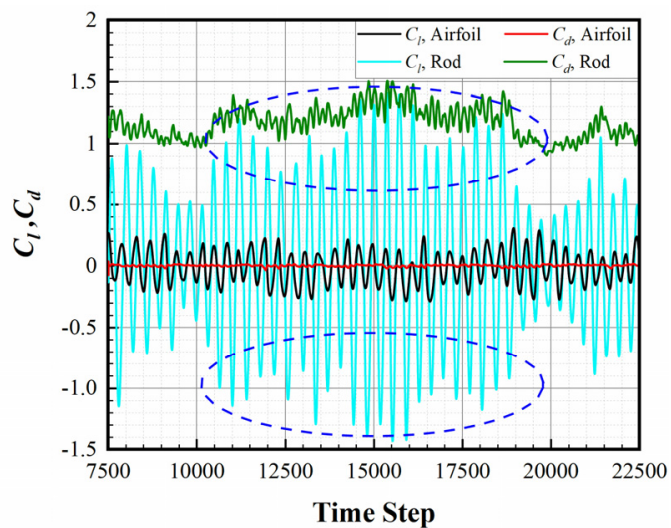

(a)

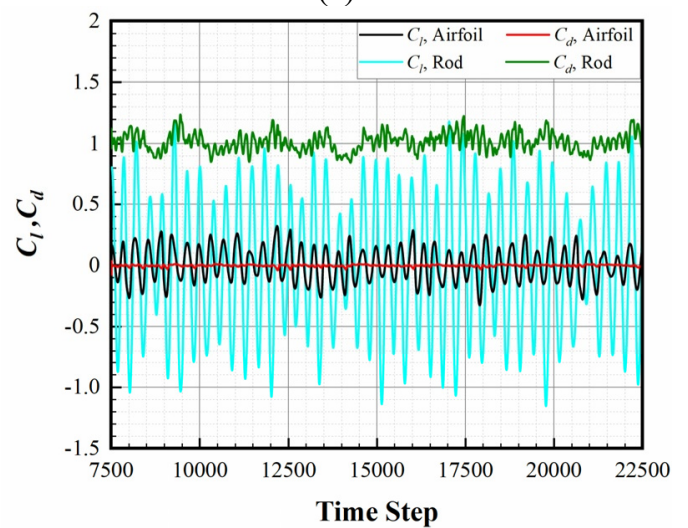

(b)

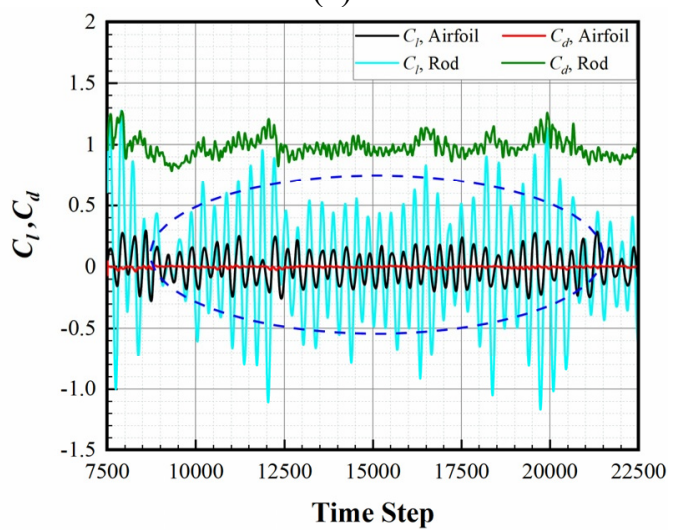

(c)

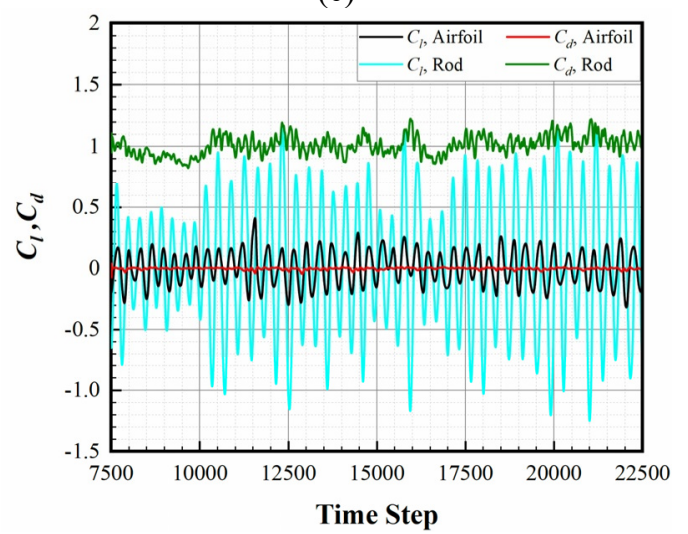

(d)

Fig. 3. The time histories of lift and drag coefficients for rod and airfoil under different subgrid-scale models. 
Table 1. Different subgrid-scale model and results on the rodairfoil

\begin{tabular}{|c|c|c|}
\hline Method & St & $\begin{array}{c}\text { Relative } \\
\text { error }\end{array}$ \\
\hline Experiment [8] & 0.192 & - \\
\hline SL & 0.1892 & $-1.46 \%$ \\
\hline DSL & 0.1939 & $0.99 \%$ \\
\hline WALE & 0.2081 & $8.39 \%$ \\
\hline KET & 0.1896 & $-1.25 \%$ \\
\hline
\end{tabular}

The SPL of the four subgrid-scale models at $1.85 \mathrm{~m}$ for the angular position $90^{\circ}$ is depicted in Figure 4. There is concern that the peak values of SPL of the SL model shows an abnormal phenomenon of "double peak" (dark blue curve); this may be because the $C_{s}$ of the SL model is selected according to experience, and there is an error between it and the dynamic changing flow field, which does not reflect the energy transfer effect. Therefore, the SPL of a certain frequency is predicted too high. The result of DSL model is in good agreement with the experimental value. The comparison results from this SPL can prove again the necessity of selecting the most suitable subgrid-scale model for a particular problem. Based on the previous analysis and considering the calculation accuracy on the flow field and the acoustic field, the DSL subgrid-scale model is finally selected for further study on identification of rodairfoil interaction noise source. Different acoustic integral surfaces are used to study the sound sources as shown in Figure 5. Surface 1 (S1) and Surface 2 (S2) are the rod wall surface and airfoil wall surface, respectively; Surface $3(\mathrm{~S} 3)$ is the permeable data surface surrounding the rod, and Surface 4 (S4) is the permeable data surface surrounding the airfoil. S1 and S2 can obtain the dipole sound sources on the rod and the airfoil surfaces, whereas S3 and S4 can obtain dipole sources as well as the quadrupole sources surrounded by them.

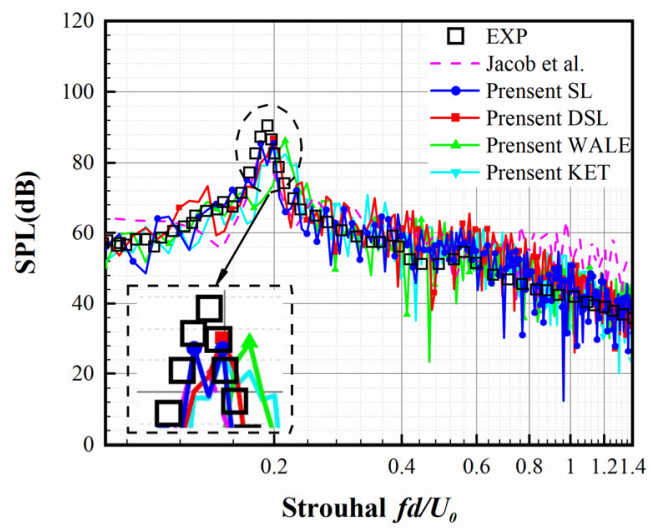

Fig. 4. Comparison of SPL of four subgrid-scale models at $90^{\circ}$ and at $1.85 \mathrm{~m}$

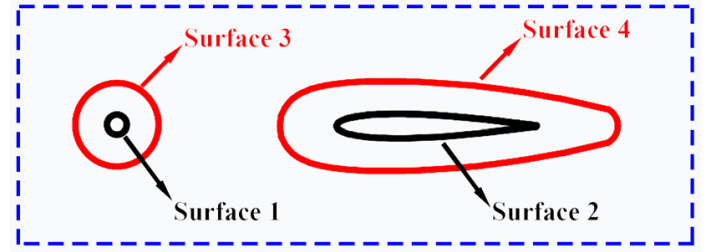

Fig. 5. Sketch of acoustic integral surfaces.

Figure 6 shows prediction results of SPL at the different acoustic integral surfaces. It is observed in Figure 6 (a) that the noise of the all-frequency at airfoil is larger than that of the rod, and the quadrupole noise produced by the rod and the airfoil can be ignored at the all-frequency. In Figures 6 (b) and (c), compared with the airfoil noise, the rod noise can be ignored, and the airfoil noise is the main sound source. Moreover, it can be seen from Figure 16 (d) that the noise generated by the solid wall S1 and S2 is basically consistent with that generated by the permeable integral surface S3 and S4. And it is almost the same as the experimental results, which indicates that the quadrupole noise can be ignored relative to the dipole noise. Therefore, in the rod-airfoil interaction noise, the dipole noise from the airfoil wall is the main noise source at low Mach number, whereas the quadrupole noise from the rod and airfoil surfaces can be ignored. This will be further analyzed later.

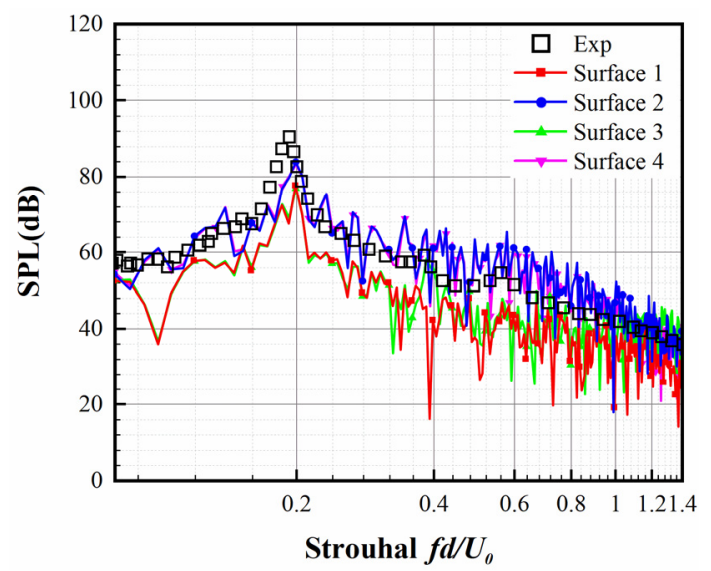

(a)

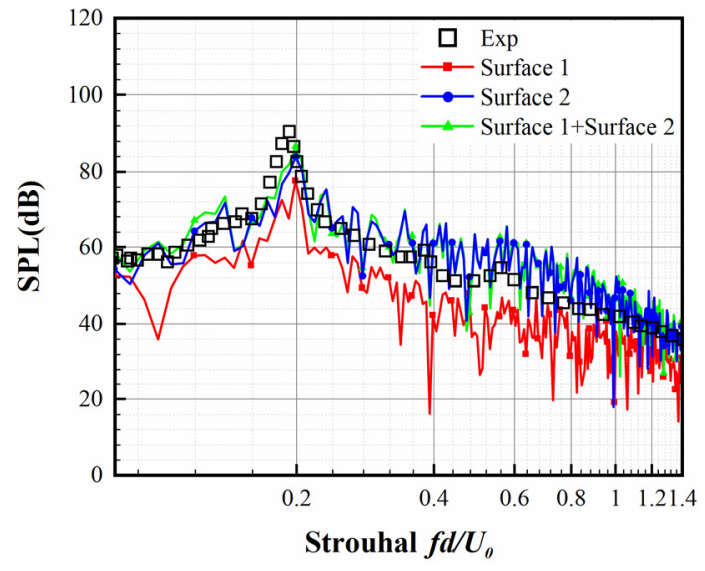

(b) 


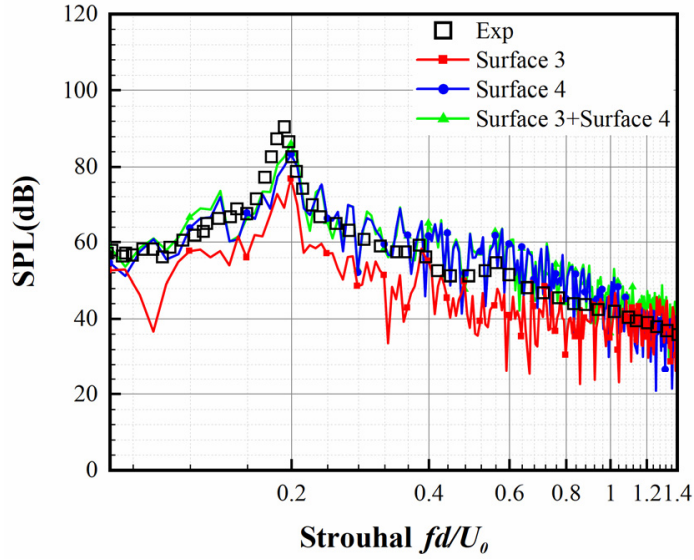

(c)

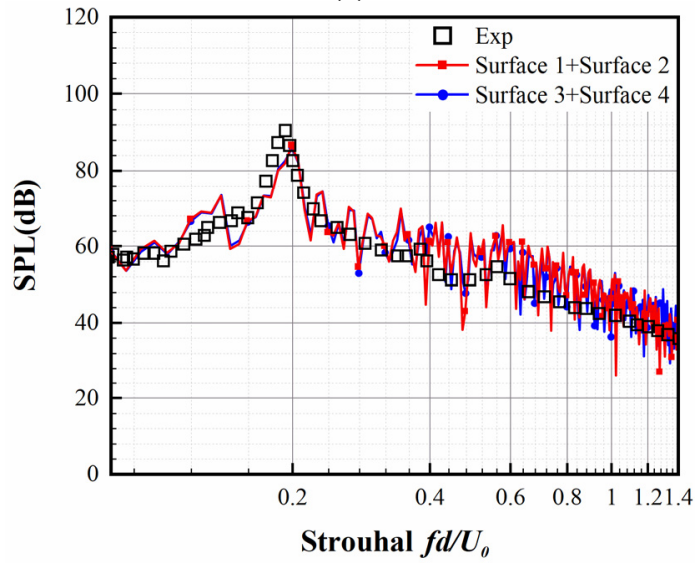

(d)

Fig. 6. Comparison of SPL at the different acoustic integral surfaces.

In Figure 7, the dipole noise produced on the airfoil wall surface is the main sound source. Since the dipole noise is produced by the pressure fluctuations on the solid wall surface, the root mean square (RMS) pressure fluctuations on the airfoil are displayed in Figure 7. In Figure 7 , the pressure fluctuations at the leading edge of the airfoil are largest, they are mainly concentrated in the range of $30 \%$ on the airfoil surface.

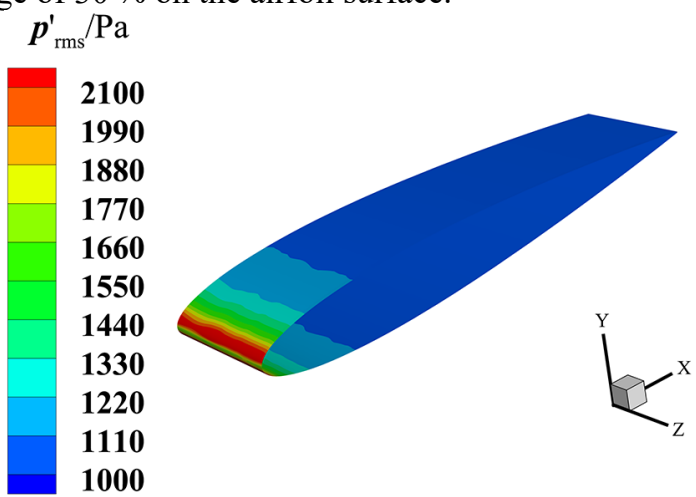

Fig. 7. RMS pressure fluctuations on the airfoil.

Figure 8 shows the SPL prediction results of the acoustic field at different integral surfaces (the entire blade surface, $30 \%$ of the airfoil leading edge, and $70 \%$ of the airfoil trailing edge), respectively. Figure 8 displays the noise generated by $30 \%$ of the airfoil leading edge is almost similar to the total noise, and it is about $9 \mathrm{~dB}$ higher than that of $70 \%$ of the trailing edge. Therefore, $30 \%$ of the airfoil leading edge is the main noise source of the overall airfoil.

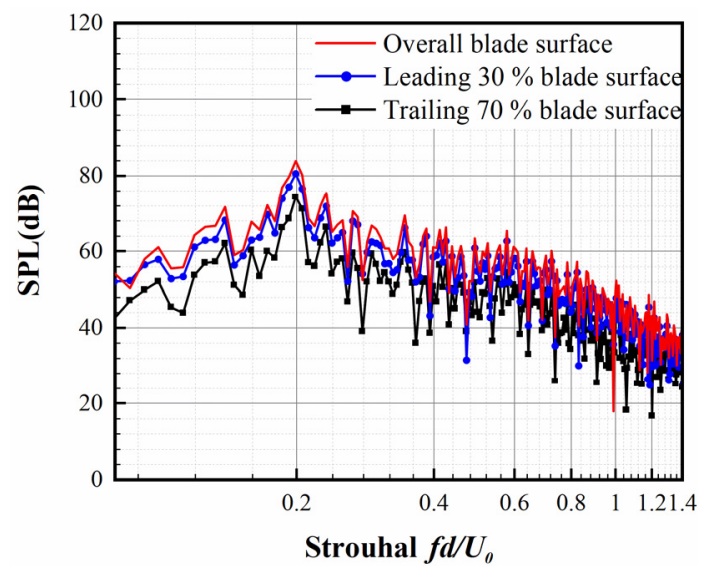

Fig. 8. Comparison of SPL at the different acoustic integral surfaces of airfoil.

\section{Conclusion}

In this paper, SL, DSL, WALE, and KET subgrid-scale models have been used to study the characteristics of rod-airfoil interaction flow field and far sound field. The DSL model, which is most suitable for the study of interaction noise, is selected by comparing it with the experimental results, and different acoustic integral surfaces are used to further identify the sound sources. In summary:

(1) The results of aerodynamic simulation and aeroacoustic prediction show that for the simulation of lift/drag coefficient, vortex shedding frequency and SPL spectrum, the results of DSL are closest to the experimental data. Therefore, it can be concluded that the DSL model is the most suitable subgrid-scale model for the research of interaction noise, and it can provide guidance for simulation research of aircraft engine rotor/stator, ventilator, helicopter blade-vortex interaction noise, etc.

(2) For the rod-airfoil interaction noise, the quadrupole noise of the whole frequency segment can be neglected at a low Mach number, and the main noise is the dipole noise caused by the unsteady pressure fluctuations on the wall surface. Compared with the noise on the cylindrical surface, the airfoil surface is the main sound source. Airfoil noise is mainly concentrated in the leading edge at $30 \%$ of the chord length. Therefore, to reduce the rod-airfoil interaction noise, it is important to reduce the dipole noise at the airfoil leading edge.

In a future study, we will consider some effective noise reduction measures, such as improving the upstream wake, boundary layer suction, and bionic modification of airfoil leading edge to investigate the rotor/stator interaction noise of aeroengine. 


\section{Acknowledgments}

The authors gratefully acknowledge the support of National Natural Science Foundation of China [grant number 51875194] and Natural Science Foundation of Hunan Province [grant number 2020JJ4306].

\section{References}

1. Wang. C, Trailing Edge Perforation for Interaction Tonal Noise Reduction of a Contra-Rotating Fan, J. Vib. Acoust. Trans. ASME, 140(2) (2018)

2. Chen. W, Qiao. W, Tong. F, Wang. L, Wang. X, Experimental Investigation of Wavy Leading Edges on Rod-Aerofoil Interaction Noise, J. Sound Vib, 422, pp. 409-431 (2018)

3. Mao. J, Zhang. K, Liu. K, Comparative Study of Different Subgrid-Scale Models for Large Eddy Simulations of Magnetohydrodynamic Turbulent Duct Flow in OpenFOAM, Comput. Fluids, 152(3), pp. 195-203 (2017)

4. Liu. C, Li. J, Bu. W, Ma. W, Shen. G, Yuan. Z, Large Eddy Simulation for Improvement of Performance Estimation and Turbulent Flow Analysis in a Hydrodynamic Torque Converter, Eng. Appl. Comput. Fluid Mech, 12(1), pp. 635-651 (2018)

5. Cheng. H. Y, Bai. X. R, Long. X. P, Ji. B. Peng, X. $X$, Farhat. M, Large Eddy Simulation of the TipLeakage Cavitating Flow with an Insight on How Cavitation Influences Vorticity and Turbulence, Appl. Math. Model, 77, pp. 788-809. (2020)

6. Shi. L, Wang. Y, Jin. Y, Zhang. G, Zhang. D, Parametrical Study and Turbulence Analysis of High-Speed Flows around an Open Cavity Using Large Eddy Simulation, Fluid Dyn. Res, 51(3) (2019)

7. Casalino. D, Jacob. M, Roger. M, Prediction of Rod-Airfoil Interaction Noise Using the FfowcsWilliams-Hawkings Analogy, AIAA J, 41(2), pp. 182-191 (2003)

8. Jacob. M. C, Boudet. J, Casalino. D, Michard. M. A, Rod-Airfoil Experiment as Benchmark for Broadband Noise Modeling, Theor. Comput. Fluid Dyn, 19, pp. 171-196 (2005)

9. A. Mecke, I. Lee, J.R. Baker jr., M.M. Banaszak Holl, B.G. Orr, Eur. Phys. J. E 14, 7 (2004) 